\title{
تطوير كتاب تعليم اللغة العربية في ضوء المدخل السياقي في المدرسة الثانوية الإسلامية الحكومية الثانية بفري فاري سولاويسي الجنوبية.
}

\author{
Andi Arief Pamessangi \\ Email: ariefpamessangi@yahoo.co.id \\ IAIN Palopo
}

\begin{abstract}
The purpose of this research is 1) to developing the Arabic learning book based on Context Approach in Islamic Senior High School (MAN) II Parepare South Celebes. 2) And to know the effectiveness of this book in Arabic learning in Islamic Senior High School (MAN) II Parepare South Celebes. Researcher write this research because the needed of student in Islamic Senior High School (MAN) II Parepare South Celebes to learning book and learning materials that's relate with them social and culture context. And the reason of this problem because in there is not another learning book and learning materials, but the materials of Arabic learning just from a learning book that develop based on 2013 curriculum that using in this school.Beside that theory of process developing a learning book have to relate with students ability and them social culture context, because the social culture context is a basic of developing the learning book.This research uses Research and Development method $(R \& D)$. In this research, researchers found 1) the Arabic learning book based on Context Approach in Islamic Senior High School (MAN) II Parepare South Celebes, that have characteristic; a) developing based on student context that is them social and culture, b) the materials learning consider of language elements, that is vocabulary and syntax, and then language ability, that is listening, speaking, reading, and writing. c) examples of this book is the student daily context. d) exercise of this book developing based on student daily context. 2) using of Arabic learning book that be developed based on Context Approach is effective in Arabic learning in Islamic Senior High School (MAN) II Parepare South Celebes. That is seeing in the result of trying in experiment class and control class with the tresult 3,234 > t table in 5\% (2,719) and $1 \%(2,074)$.
\end{abstract}

Keywords: Developing, Material of Arabic Learning, Context Approach 


\section{مستخلص البحث}

أهداف هذا البحث 1) لإنتاج كتاب تعليم اللغة العربية في ضوء المدخل السياقي في المدرسة الثانوية الإسلامية الحكومية الثانية بفري فاري سولاويسي الجنوبية. و 2) لقياس فعالية تعليم اللغة العربية باستخدام كتاب تعليم المطور في ضوء المدخل السياقي في المدرسة الثانوية الإسلامية الحكومية الثانية بفري فاري سولاويسي الجنوبية. يقوم الباحث بهذا البحث بسبب إحتياجات الطلبة في المدرسة الثانوية الإسلامية الحكومية الثانية بفري فاري سولاويسي الجنوبية إلى كتاب ومواد تعليم اللغة العربية المناسبة

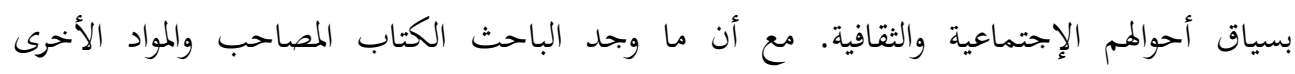
المستخدمة في تعليم اللغة العربية في هذه المدرسة الا بمواد التعليم الموجودة في كتاب تعليم اللغة العربية بالمنهج 2013. وبجانب ذالك في نظرية تطوير مواد وكتاب التعليم ينبغي على المدرس أن يختار مادته العلمية واللغوية وفق بمعايير وأسس علمية. ولن يمقق الكتاب المدرس أهدافه ما لم تكن مادته اللغوية في متناول قدرات الدارسين العقلية وخلفياقم الثقافية لأن الثقافة والإجتماعية هي إحدى الأساس من أسس إعداده. ولذلك يطور الباحث كتاب التعليم في ضوء المدخل السياقي في المدرسة الثانوية الإسلامية الحكومية الثانية بفري فاري سولاويسي الجنوبية، وقياس فعاليته في تعليم اللغة الربية في هذه المدرسة. ومدخل البحث هو مدخل البحث والتطوير ـ وملخص نتائج البحث هو، 1 ) ينتج الباحث كتاب تعليم اللغة العربية في ضوء المدخل السياقي في المدرسة الثانوية الإسلامية الحكومية الثانية بفري فاري سولاويسي الجنوبية، بالمواصفات؛ أ) تألف هذا الكتاب حسب الموضوعات التعليمية لتعليم اللغة العربية في ضوء المدخل السياقي. والمواصفات المحلية هي الموضوعات المحلية والصور التي تتعلق بثقافة فري فاري. ب)يتكون هذا الكتاب من: مواد عناصر اللغة وهي المفردات التي تتعلق بأحوال الطلبة اليومية والتركيب. ومواد مهارات اللغة وهي الإستماع، والكلام، والقراءة، والكتابة. ج) تقدم الأمثلة المتعلقة بأحوال الطلبة د) التدريبات المتنوعة المناسبة بمواد التعليم وأحوال الطلبة. ونتائج البحث الثاني هي 2) الكتاب التعليمي الجديد الذي أعده الباحث في ضوء المدخل السياقي له فعالية في تنمية معرفة اللغة العربية لطلبة الفصل العاشر في المدرسة الثانوية الإسلامية الحكومية الثانية بفري فاري سولاويسي الجنوبية. يبدو ذلك عندما 
قارن الباحث بين النتائج التي حصل عليها طلبة المجموعة التجربة وطلبة البمموعة الضابطة، وذلك أن درجة تاء حساب (t) في هذا البحث (234،3) أكبر من درجة تاء الجدول على مستوى 50 (2،719، و من درجة تاء الجحدول على مستوى 1\% (2) (20) (074).

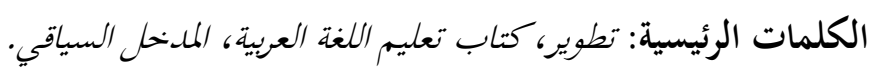

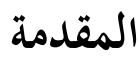

مشكلة كتاب التعليم كثيرة ومتنوعة، منها إختلاف محتويات الكتاب مع حاجة الطلبة، كما قال ستي روح الله أن عملية التعليم تحتاج إلى كثير من المواد الجذابة والمثيرة للاهتمام، لأن المواد من المكونات المهمة في العملية التعليمية، وهي إذ لم تعد إعدادا جيدا ويتم اختيارها وفقا بالإحتياجات فقد لا تأتي بالفائدة المرجوة للطلبة ولا تساعد المدرسين على الوصول إلى الأهداف المرجوة. 1 المشكلة الأساسية للكتاب التعليمي هي محتوياته لا تشتمل بأحوال الطلبة في حياهم اليومية. فلازم على المدرس أن يعد الكتاب التعليمي المناسب بحاجة الطلبة وكذلك مناسب بأحوالهم. المدخل المناسب بتلك المشكلة هو المدخل السياقي. وهو يصدر من مفهوم الفلسفي جون دي الذي يركز التعليم على ميول الطلبة وخبرةم وخلفياهم. التعليم على السياق العام هو الإبتاه في عملية التعليم التي

1 ستي روح الله، إعداد المواد التعليمية من الثقافة المحلية لتنمية مهارة الكلام، رسالة الماجستير، (مالنج: جامعة مولانا مالك ابراهيم الإسلامية الحكومية 
تعلق المواد التعليمية بأحوال الطلبة وحياتم وثقافتهم حتى تكون الدراسة ذات

$$
\text { علاقة وثيقة بأحوالهم وذات معنى قوي في ذهنهم. } 2
$$

المدرسة الثانوية الإسلامية الحكومية 2 (الثانية) بفري فاري سولاوسي الجنوبية

هي من إحدى المدارس المشهورة في مدينة فري فاري، والكتاب التعليمي المستخدم في تعليم اللغة العربية فيها هي كتاب دروس اللغة العربية على منهج 2013 لوزارة الدينية لإندونيسية. وأهم المشكلة التي يجدها الباحث في ميدان البحث هي عدم علاقة بين محتويات كتاب تعليم اللغة العبية مع أحوال الطلبة، المحتويات لا تشتمل بأحوال الطلبة حتى لا يستطيعون أن يستخدمو اللغة العربية في حياتم اليومية. وهذا بسبب كتاب دروس اللغة العربية على منهج 2013 يهدد على الأحول الإجتماعية والثقافية بإندونيسيا عامة فأحسن للمدرس أن يطور كتاب تعليم اللغة العربية المناسب بأحوال الحناصة للطلبة في هذه المدرسة. مع أن من أهداف تعليم اللغة العربية في منهج 2013 هي التمنية لكي يفهم الطلبة قيمة الثاقفة من محتويات المواد التعليمية ويتطبقو معرفتهم عن اللغة العربية مناسبا بسياق أحوالهم النفسية والإجتماعية والثقافية. 3

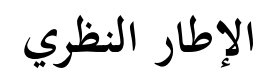
تطوير كتاب التعليم

\footnotetext{
${ }^{2}$ Kesuma Dharma, dkk, Contextual Teaching and Learning. (Garut: Rahaya Research and Training, 2010). Hal.5..

${ }^{3}$ Lampiran SK DIRJEN No. 2676 Tahun 2013 tentang Kurikulum 2013 Mata Pelajaran Pendidikan Agama Islam Dan Bahasa Arab Di Madrasah. hal.224.
} 
إن مصطلح الكتاب المدرسي يختتلف عن المعنى الشائع المألوف لهذا المصطلح، أن يتألف الكتاب المدرسي لا من مادته الأساسية فحسب، بل كذلك من المواد المساعدة كالمعجم وكتاب التمرينات التحريرية أو الصوتية وكتب المطالعة المتدرجة وكتاب الاختبارات ومرشد المعلم. 4 ويلزم عند إعداد وتطوير الكتاب لتعليم اللغة العربية لناطقين بغيرها اهتمام الأسس وهي الأسس الثقافية والإجتماعية والأسس السيكولوجية والأسس الغوية والتربوية. ${ }^{5}$ وفق على هذا الرأي ، قدم محمود كامل الناقة ورشدي أحمد طعيمة الأسس أو الجوانب المهمة في إعداد كتاب التعليم وهي الجانب النفسي والجانب الثقافي والجانب التربوي والجانب اللغوي. وينبغي أن نختار مادة الكتاب وفق معايير وأسس علمية. ولن يهقق محتوي الكتاب أهدافه مالم تكن مادته اللغوية في متناول قدرات الدارسين العقلية وخلفياهم الثقافية. 6 اما المعايير في إختيار محتوى الكتاب لا بد من الإهتمام بها، منها؛ معيار الصدق (validity)، ومعيار الأهمية (significance)، ومعيار الميول والإهتمامات

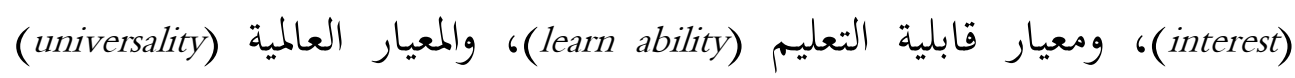

$$
\begin{aligned}
& \text { 4 ناصر عبد الله الغالي وعبد الحميد عبد الله، أسس إعداد الكتب التعليمية لغير الناطقين بها، (الرياض: دار الغالي. 1991)، ص. } 9 .
\end{aligned}
$$

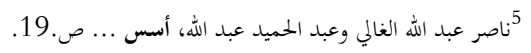

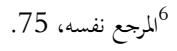


7. وهناك عدة أساليب يمكن لواضع المنهج اتباعها عند اختيار مواد اللغة العربية. لغير الناطقين بها؛ المناهج الأخرى، ورأي الخبير، والمسح، والتحليل. 8 وهناك التصوران لتنظيم المحتوى، الأول التنظيم المنطقي وهو تقديم المحتوى مرتبا في ضوء المادة ذاتا، أي مراعات الترتيب المنطقي للمعلومات والمفاهيم بصرف النظر عن مدى قالبة الطلبة مثل تبدأ الموضوعات من موضوع بسيطة إلى موضوعة معقدة، من السهل إلى الصعب، من القديم إلى الحديث. والثاني التنظيم السيكولوجي وهو تقديم المحتوى في ضوء إحتياجات الطلبة. 9 والمعايير في تنظيم المحتوى ثلاثة وهي الإستمرارية والتتابع والتكامل. واما أسس تأليف الكتاب هي:

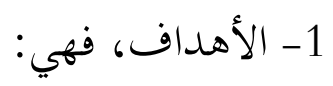

- المعريفية، وهي المادة المغزية التي يلزم للطلبة أن يعلموها

- - المهارية، وهي القدرة العملية التي تطور من المعلومات وتصير مطبقة - - السلوكية، وهي السلوك التي يجب للطلبة أن يملكوها 2- إعداد محتوى الكتاب المقرر، وهي: - مضبوط، أن يكون المحتوى مضبوطا من الناحية العلمية

\footnotetext{
7إبراهيم بسوني عميرة، المنهج وعناصره، الطبعة الثالثة (القاهرة: دار المعارف،1991)ص:160.

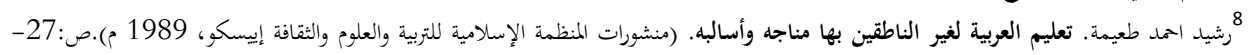

"المرجع نفسه...ص:69 
91 | تطوير كتاب تعليم اللغة العبية في ضوء المدخل السياقي في الملرسة الثانوية ... - مناسب، أن يكون المحتوى مناسبا بالكفاءة الرئيسية والكفاءة

$$
\begin{aligned}
& \text { الأساسية } \\
& \text { - وافي، أن يكون المحتوى لا كثير ولا قليل. } \\
& \text { - مطابق، تنوع المحتوى وكثرته مناسبان بالكفاءة } \\
& \text { - واقعي، أن يناسب المحتوى بتطوير العلوم والتكنولوجي }
\end{aligned}
$$

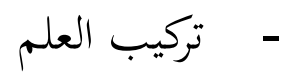

$$
\begin{aligned}
& \text { 3- اللغة والمقروئية، وهي: } \\
& \text { - - استعمال الجمل مناسب بالقواعد اللغوية الصحيحة } \\
& \text { - يدل ترتيب الجمل على تفكير معقول ونظامي } \\
& \text { - ترتيب الجمل مناسب لقدرة لغة الطلبة } \\
& \text { - }
\end{aligned}
$$

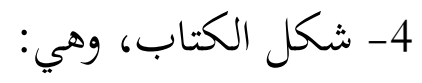

$$
\begin{aligned}
& \text { - - أن تصميم حجم الكتاب ومكان شرح المحتوى والصور مناسبة } \\
& \text { - }
\end{aligned}
$$$$
\text { طرق تعليم اللغة العربية للناطقين بغيرها وموادها }
$$

\footnotetext{
${ }^{10}$ Kokom Komalasari, Pembelajaran Kontekstual Konsep dan Aplikasi, (Bandung: PT.Refika Aditama, 2010) hal. 46-48 .
} 
إن طريقة التعليم تعني الخطة الشاملة التي يستعان بها في تحقيق الهدف التربوي المنشود. 11 وأهم أنواع طرق التدريس في اللغة الغربية لغير الناطقين بها هي؛ طريقة القواعد والترجمة وطريقة المباشرة وطريقة القراءة والطريقة السمعية الشفهية والطريقة التوليفية. 12

وأما المواد التعليمية للغة العربية هي المحتوي التعليمي لهذه اللغة الذي يرغب معلمها في تقديمه للطلبة بغرض تحقيق أهداف تعليمية معرفية أو مهارية أو وجدانية. وهي المضمون الذي يتعلمه الطلاب في علم ما. وهي مصدرا أساسيا في تعليم اللغة العربية إما من شكل النص أو غيره والأشياء التي يتعلمها الطلبة أو يعلم إليهم.

وقد قدم رشدي أحمد طعيمة عن بعض الموضوعات الثقافية في تعليم اللغة العربية للأجانب، منها البيانات الشخصية، والسكن، والعمل، ووقت الفرغ، السفر، والعلاقات مع الآخرين، والصحة والمرض، والتعليم والتربية، ويف السوق، وفي المطعم، والخدمات، والبلدان والأماكن، واللغة الأجنبية، والجو، والمعالم الحضارية، والحاية الإقتصادية، والدين والقيم والروحية، والسياسة، والعلاقات

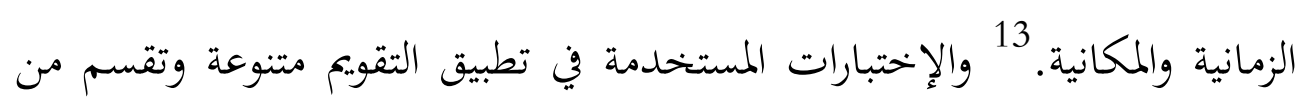

$$
\text { 11 بردي أممد طعيمة، المرجع...ص.203. }
$$

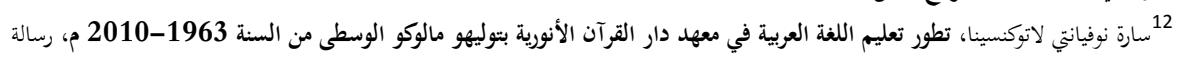

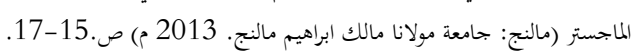

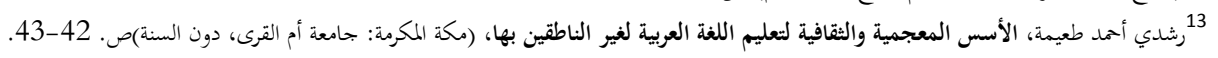


جهة مواصفتها إما من جهة أهدافها وكيفية قيامها وشكل جوابها وطريقة تقويمها وقت قيامها وإستفادتها وغيرها. 14 وقد بيّن إمام اسراري وآخرون على هذه الأنوع في كتابهم تقويم تعليم اللغة العربية:

1) من جهة كيفية قيامها؛ الإختبار التحريري والإختبار الشفهي

2) من جهة شكل الجواب؛ الإختبار المقال والإختبار الإختيار

3) من جهة طريقة تقويمها؛ الإختبار الموضوعي والإختبار الذاتي

15

4) من جهة وقت قيامها؛ الإختبار التكويني والإختبار الختامي/ النهائي. واما بجالات الاختبار الخاصة للغة العربية تتكون من:

$$
\begin{aligned}
& \text { - الاختبار للعناصر اللغوية } \\
& \text { - } \\
& \text { اهداف تعليم اللغة العربية في منهج } 2013
\end{aligned}
$$

إن الغرض الأساسي من تعليم اللغة العربية هو أن يستطيع الطالب أن يعتبر

عن نفسه تعبيرا كاملا صحيحا باللسان أو القلم وأن يفهم ما يقرأ أو ما يسمع، وأن يشارك في التفكير فيما حوله بقدر ما تسمح به سنه ومواهبه. 17 وأما أهداف التعليم اللغة العربية على المنهج 2013 هي التمنية لكي يفهم الطلبة قيمة الثاقفة من محتويات المواد التعليمية هي إحدى الهدف من أهداف تعليم اللغة العربية على

\footnotetext{
${ }^{14}$ Abdul Hamid, Mengukur Kemampuan Bahasa Arab,(Malang: UIN Maliki Press,2013), hal.12.

${ }^{15}$ Imam Asrori, dkk. Evaluasi Pembelajaran Bahasa Arab, (Malang: Misykat, 2014), hal. 84.

hal.96... ${ }^{16}$ Imam Asrori, dkk. Evaluasi 17 17 17
} 
هذا المنهج. وكذلك لكي يطبقو معرفتهم عن اللغة العربية مناسبا بسياق أحوالهم النفسية والإجتماعية والثقافية هي أيضا من إحدى هدفه. 18

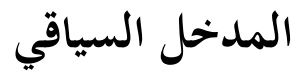

المدخل بفتح الميم هو الدخول او بمعنى موضع الدخول. 19 مدخل التعلم هو

وسيلة أو وسائل تستخدمها المعلمون لتمكين الطلبة أن يتعلموا. هو وسيلة مشتركة للنظر مشكلة أى موضوء الدراسة. 20 المدخل السياقي يركز التعليم على ميول الطلبة وخبرةم وخلفياتم. وهو المدخل الذي يحاول مساعدة الطلبة في نظر المواد التعليمية المتعلمة ويعلقها بأحوالهم اليومية إما من الجهة الشخصية أو الإجتماعية أو الثقافية. 21 مطابقا بها يرى كسوما أن التعليم على السياق هو الإبتاه في عملية التعليم التي تعلق المواد التعليمية بأحوال الطلبة وحياهم وثقافتهم حتى تكون الدراسة ذات علاقة وثيقة بأحوالهم وذات معنى قوي في ذهنهم. 22 أسس هذا المدخل سبعة اسس، وهي التركيب، والاستعلام، والتسائل، تعلم الجماعة، تحضير النموذج، الإنعكاس، والتقويم الذاتي.

\footnotetext{
${ }^{18}$ Lampiran SK DIRJEN no. 2676 Tahun 2013 tentang Kurikulum 2013 Mata Pelajaran Pendidikan Agama Islam dan Bahasa Arab di Madrasah. hal.. 220-225.

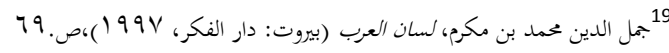

${ }^{20}$ Ujang Sukandi dkk,Belajar Aktif dan terpadu (Surabaya:Duta Graha Pustaka,2003).hal.39.

${ }^{21}$ Johnson dalam, Nurhadi, dkk. Pembelajaran Kontekstual (Contextual Teaching and
} Learning/CTL) dan Penerapannya dalam KBK. (Malang: Universitas Negeri Malang 2002 ).

Hal.12.

${ }^{22}$ Kesuma Dharma, dkk, Contextual Teaching and Learning. (Garut: Rahaya Research and Training, 2010). Hal.5. 
95 | تطوير كتاب تعليم اللغة العربية في ضوء المدخل السياقي في الملرسة الثانوية ....

$$
\text { أسلوب جمث البحث البيانات وتحليلها }
$$

ليحصل على الإنتاج الجيد، فاستعمل الباحث ثلاث خطوات وهي : 1) تحليل الحاجات قبل عملية الإعداد. 2) نيل المداخلات والإقتراحات من خبراء هذا البحث، وهما : أ) الخبير في تطوير مواد اللغة العربية، ب) الخبير في المدخل السياقي • 3) معرفة فعالية الإنتاج لتنمية معرفة اللغة العربية للطلبة. وفي جمع البينات استخدم الباحث؛ الملاحظة، يستعملها الباحث لجمع البييانات المتعلقة بالمنهج، والكتاب المستخدم في تعليم اللغة العربية خاصة لاللغة العربية في الفصل العاشر في هذه المدرسة. والمقابلة، يستخدمها الباحث لجمع البيانات عن معلومات من مواد اللغة العربية والكتاب والطريقة والوسائل والإختبرات المستخدم في التعليم. والإستبانة، يوجهها الباحث المى الخبيرين ومدرسة اللغة العربية للحصول على كتاب التعليمي المناسب، ويوجه الى الطلبة لمعرفة رأيهم عن التعلم بعد ما درسوا بالكتاب المطور. والإختبار، وهما الإختبار القبلي والإختبر البعدي. والوثائق، استخدمه الباحث لتحليل المنهج الدراسي وكذلك محتوى سياق مدينة فري فاري الثقائ والإجتماعي والمصادر المتعلقة بتطوير كتاب التعليم. 
ويحلل الباحث البيانات بطريقتين وهي تحليل البيانات النوعية يعني قام الباحث بتحليل البيانات من المقابلة والملاحظة والوثائق، يستخرجها الباحث جميع البيانات الموجودة ثم تحررها وترتبها وتعرضها وتحللها بالاستنتاج. تثم تحليل البيانات الكمية

$$
\begin{aligned}
& \text { من الإستبانة و من الإختبار. } \\
& \text { نتائج البحث }
\end{aligned}
$$

وينتج الباحث كتاب تعليم اللغة العربية في ضوء المدخل السياقي في المدرسة

الثانوية الإسلامية الحكومية الثانية بفري فاري سولاويسي الجنوبية، بالمواصفات؛ أ) تألف هذا الكتاب حسب الموضوعات التعليمية لتعليم اللغة العربية في ضوء المدخل السياقي. والمواصفات المحلية هي الموضوعات المحلية والصور التي تتعلق بثقافة فري فاري. ب)يتكون هذا الكتاب من: مواد عناصر اللغة وهي المفردات التي تتعلق بأحوال الطلبة اليومية والتركيب. ومواد مهارات اللغة وهي الإستماع، والكلام، والقراءة، والكتابة. ج) تقدم الأمثلة المتعلقة بأحوال الطلبة د) التدريبات المتنوعة المناسبة بمواد التعليم وأحوال الطلبة. الكتاب التعليمي الجحديد الذي أعده الباحث في ضوء المدخل السياقي له فعالية في تنمية معرفة اللغة العربية للطلبة. يبدو ذلك عندما قارن الباحث بين النتائج التي حصل عليها طلبة المجموعة التجربة وطلبة البحموعة الضابطة، وذلك أن درجة تاء حساب (to) في هذا البحث 
(234،3) أكبر من درجة تاء الجمدول على مستوى 5\% (2،719) و من درجة تاء

$$
\text { الجحدول على مستوى 1\% (2، 074). }
$$

الخحاتمة

التوصيات

فمن الحسن أن تقوم المدرسة بإعداد المواد التعليمية الجيدة، وذلك باختيار

الموضوعات السهلة والجوابة وما لها ارتباطب أحوالهم اليومية حتى لا يمل منه الطلبة. وأن تستخدم المدرسة أساليب التدريس والنشاطات التعليمية المتعددة ويستعين بالوسائل المعينة المتنوعة وأساليب التقويم المختلفة حتى يكون التعليم عملية مريحة غير محلة. وأن تزود المدرسة أنواع التدريبات لإثراء معرفة الطلبة وتنويع خبراهم وتوثيق كفاءهم. وينبغي أن بتتنب المدرسة استخدام لغة الأم عند تدريس اللغة العربية،لأن ذلك يجعل الطلبة يتعود ونعلى ترجمة الدرس أو شرحه بلغة الأم، بل تلزم عليها استخدام كثير من الوسائل المعينة لفهم معاني المفردات التي يريد تعليمها. المقترحات

بناء على ما جاء في هذا البحث، يقدم الباحث بعض المقترحات منها: 1) إن كتاب تعليم اللغة العربية المطور في ضوء المدخل السياقي أداة من الأدوات المهمة في العملية التعليمية التي يمكن أن يستفيد منها المدرسة اللغة العربية 
لطلبة فصل العاشر بالمدرسة الثانوية الإسلامية الحكومية الثانية بفري فاري

$$
\text { سولاويسي الجنوبية. }
$$

2) ويرجى من الباحثين الآخرين أن يطوروا المواد التعليمية في تعليم اللغة العربية من البيئة التي يعيش فيها الطلبة ومتعلقة بالأمور حولهم، حتى لا تؤدي المواد إلى وجود فجوة بين المتعلمين والمواد التي بين أيديهم. إن كتاب تعليم اللغة العربية المطور في ضوء المدخل السياقي الذي طوره الباحث في هذا البحث مركّز على تطوير كتاب التعليم لطلبة فصل العاشر نصف السنة الأولى بالمدرسة الثانوية الإسلامية الحكومية الثانية بفري فاري سولاويسي الجنوبية، فيحسن أن توجد بجوث أخرى لتطوير كتاب التعليمي لنصف السنة و الفصول

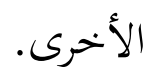

\section{قائمة المراجع والمصادر}

جمل الدين محمد بن مكرم، لسان العرب. بيروت: دار الفكر، و998 19 .

الغالي، ناصر عبد الله وعبد الحميد عبد الله. أسس إعداد الكتب التعليمية لغير الناطقين بها، الرياض: دار الغالي، 1991م

طعيمة، رشدي أحمد. تعليم العربية لغير الناطقين بها مناجه وأسالبه. منشورات المنظمة الإسلامية للتربية والعلوم والثقافة ، إييسكو، 1989 م. 
99 | تطوير كتاب تعليم اللغة العربية في ضوء المدخل السياقي في الملرسة الثانوية ....

طليمات، غازي مختار. علم اللغة، ط.2 ـدمشق: دار طلاس للدراسات والترجمة

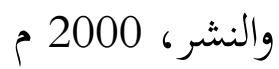

عثمان، عبد الرحمن أحمد. مناهج البحث العلمي وطرق كتابة الرسائل الجامعة. جامعة إفريقيا العالمية، 1995 م

عميرة، إبراهيم بسوني. المنهج وعناصره، الطبعة الثالثة. القاهرة: دار

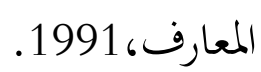

روح الله، ستي. إعداد الكتاب التعليمي من الثقافة المحلية لتنمية مهارة الكلام، رسالة الماجستير. مالنج: جامعة مولانا مالك ابراهيم الإسلامية

$$
\text { الحكومية مالنج.2012. }
$$

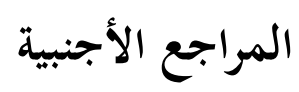

Asrori, Imam, Muhammad Thohir. M.Ainin. Evaluasi Pembelajaran Bahasa Arab. Cet.4. Malang: Misykat, 2014.

Dharma, Kesuma, dkk. Contextual Teaching and Learning. Garut: Rahaya Research and Training, 2010.

Hamid, H.M.Abdul. Mengukur Kemampuan Bahasa Arab. Malang: UIN Maliki Press,2013.

Koentjaraningrat. Pengantar Antropologi I. Jakarta: Rineka Cipta,2003.

Nurhadi, dkk. Pembelajaran Kontekstual (Contextual Teaching and Learning/CTL) dan Penerapannya dalam KBK. Malang: Universitas Negeri Malang. 2002.

Sukandi, Ujang dkk, Belajar Aktif dan terpadu. Surabaya:Duta Graha Pustaka, 2003. 